\title{
Surgical navigation for challenging recurrent or pretreated intra-abdominal and pelvic soft tissue sarcomas
}

\author{
Sophie J. M. Reijers MD ${ }^{1}$ (1) | Wouter J. Heerink PhD ${ }^{1}$ | Ruben Van Veen ${ }^{1}$ | \\ Jasper Nijkamp PhD ${ }^{1}$ ～Nikie J. Hoetjes ${ }^{1}$ | Yvonne Schrage MD, PhD $^{1}$ | \\ Alexander Van Akkooi MD, PhD ${ }^{1}$ | Geerard L. Beets MD, PhD ${ }^{1}$ \\ Frits Van Coevorden MD, PhD ${ }^{1}$ | Theo J. M. Ruers MD, $\mathrm{PhD}^{1,2}$ | \\ Harald C. Groen PhD ${ }^{1}$ | Winan J. Van Houdt MD, MSc, PhD ${ }^{1}$
}

\footnotetext{
${ }^{1}$ Department of Surgical Oncology, The Netherlands Cancer Institute/Antoni van Leeuwenhoek Hospital, Amsterdam, The Netherlands

${ }^{2}$ Faculty of Science and Technology (TNW), Nanobiophysics Group, Technical University of Twente, Enschede, The Netherlands

\section{Correspondence}

Winan J. Van Houdt, Department of Surgical Oncology, The Netherlands Cancer Institute, Plesmanlaan 121, 1066 CX, Amsterdam, The Netherlands.

Email: w.v.houdt@nki.nl
}

\begin{abstract}
Background: This study assessed whether electromagnetic navigation can be of added value during resection of recurrent or post-therapy intra-abdominal/pelvic soft tissue sarcomas (STS) in challenging locations.

Materials and Methods: Patients were included in a prospective navigation study. A pre-operatively $3 \mathrm{D}$ roadmap was made and tracked using electromagnetic reference markers. During the operation, an electromagnetic pointer was used for the localization of the tumor/critical anatomical structures. The primary endpoint was feasibility, secondary outcomes were safety and usability.

Results: Nine patients with a total of 12 tumors were included, 7 patients with locally recurrent sarcoma. Three patients received neoadjuvant radiotherapy and three other patients received neoadjuvant systemic treatment. The median tumor size was $4.6 \mathrm{~cm}$ (2.4-10.4). The majority of distances from tumor to critical anatomical structures was $<0.5 \mathrm{~cm}$. The tumors were localized using the navigation system without technical or safety issues. Despite the challenging nature of these resections, $89 \%$ were $\mathrm{RO}$ resections, with a median blood loss of $100 \mathrm{ml}$ (20-1050) and one incident of vascular damage. Based on the survey, surgeons stated navigation resulted in shorter surgery time and made the resections easier.

Conclusion: Electromagnetic navigation facilitates resections of challenging lower intra-abdominal/pelvic STS and might be of added value.

KEYWORDS

electromagnetic tracking, intra-abdominal sarcoma, pelvic sarcoma, soft tissue sarcoma, surgical navigation
\end{abstract}




\section{1 | INTRODUCTION}

Soft tissue sarcoma (STS) is a heterogeneous and rare group of tumors, with over seventy different histologic subtypes, ${ }^{1}$ covering $1 \%$ of all adult malignancies. Of all STS, around 16\% arises in the retroperitoneum, intra-abdominally, or in the pelvis. ${ }^{2}$

Usually, intra-abdominal, pelvic, and retroperitoneal STS can be easily identified during laparotomy or laparoscopy, followed by resection. However, resection of pretreated tumors with systemic therapy or radiotherapy can sometimes be more challenging due to fibrosis and/or shrinkage of the tumor, while also resections of small recurrent tumors can be more difficult due to anatomical and fibrotic changes after previous surgery. ${ }^{3}$ In these situations, localization of the tumor and identification of the tumor outline during surgery can be more challenging, potentially leading to a search for the tumor in a compromised environment and an increased risk of complications. Therefore, in these cases, surgical guidance by modern navigation techniques might be useful to overcome these hurdles.

Preoperative imaging is the standard of care before any intraabdominal resection, usually by computed tomography (CT) scanning. When surgeons make a preoperative plan on how to approach the tumor and what difficulties may arise based on the imaging, they mentally transform the 2D slices into a three-dimensional (3D) surgical plan. In this modern era, innovative image-guided surgical navigation allows linking the preoperative images (and a patientspecific 3D roadmap) directly to the patient during surgery, using a tracking system. Our group has successfully implemented this technique in the OR for multiple abdominal studies. ${ }^{4-6}$ The system provides the surgeon with real-time intra-operative information on the location of the tumor(s) and critical anatomical structures. ${ }^{6}$

Theoretically, surgical navigation could shorten surgery time due to faster localization of the tumor, might make the tumor resection safer by identifying critical anatomical structures, and thereby prevent damage to vital surrounding structures. And although navigation is not designed to prevent R1 margins, it might assist in identifying the outline of the tumor and therefore decreasing R2 resections for these challenging tumors. However, surgical navigation does require additional setup time and imaging. To further analyze the potential role of surgical navigation, the aim of this study was to investigate whether electromagnetic surgical navigation is feasible and of added value during resection of challenging recurrent or pretreated intra-abdominal/pelvic STS for localization and safe removal of the tumor(s).

\section{2 | MATERIALS AND METHODS}

\section{1 | Patients}

Patients were selected from a prospective surgical navigation study (NTR 7184) carried out at the Netherlands Cancer Institute/Antoni van Leeuwenhoek hospital and approved by the institutional ethical
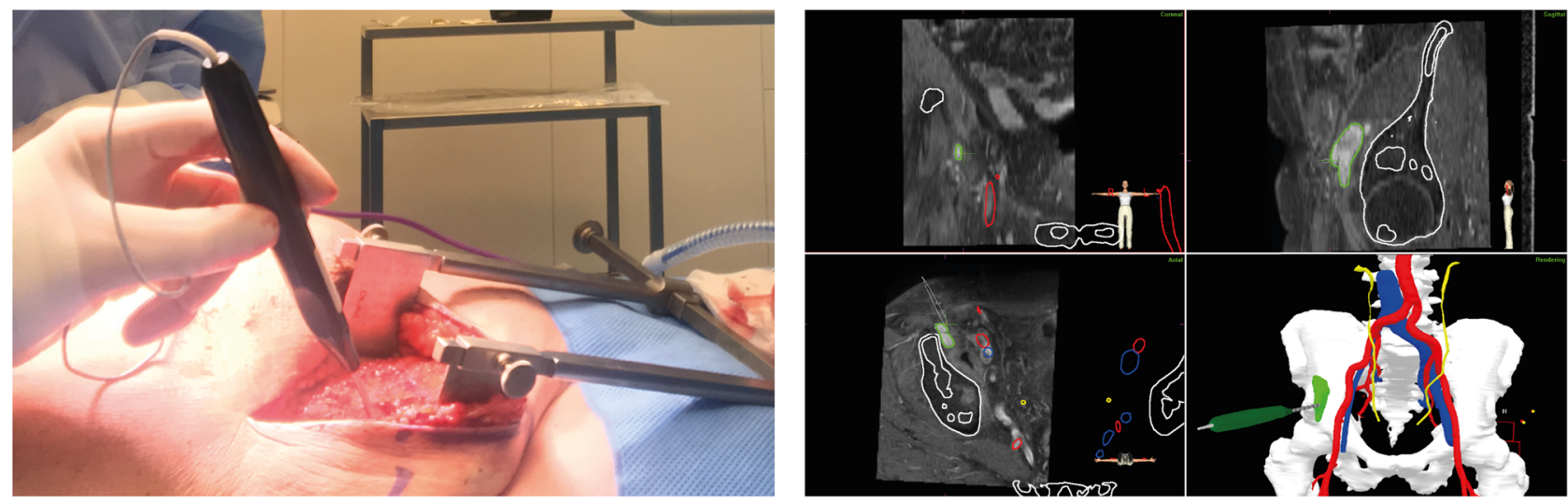

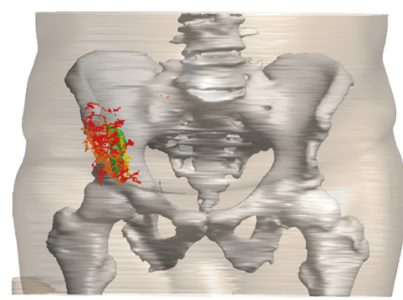

Frontal view

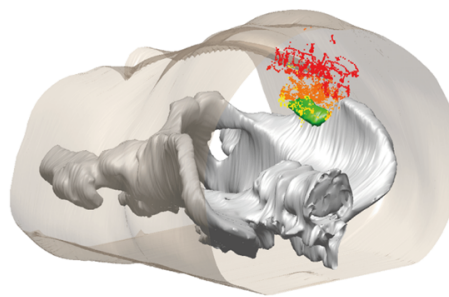

Left / top side view

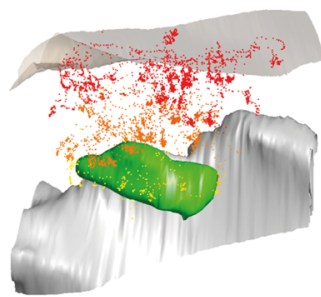

Left / top side view (zoom)

FIGURE 1 Pointer usage. Top: Real-time pointer usage visualized with in-house developed software and corresponding (3D) imaging of the patient with visualization of the tumor and critical anatomical structures. Bottom: Measuring points of the electromagnetic pointer are color-coded based on time: Early phase of surgery/superficial measurements (red), further navigation towards the tumor (orange), and final measurements before tumor resection (yellow). The tumor is marked green 
review board. Patients 18 years or older and scheduled for laparotomy with primary or local recurrent rigid tumor(s) could be included. Patients were selected on surgeons' request as the surgeons considered these patients at risk for damage to critical anatomical structures and/or the tumors were located in a difficult anatomical location, or when the surgeon felt that pretreatment might compromise identification of the tumor. For the original study, there were no restrictions based on tumor type, providing all tumors were located in the abdomen. For this analysis, we selected only STS patients. Part of these patients was already presented in the general surgical navigation feasibility study by Nijkamp et al. ${ }^{6}$ Tumor rigidity was assessed by imaging and based on location, the extent of local invasion or tumor displacement relative to bone structures between follow-up imaging. Exclusion criteria were contraindications for intravenous CT contrast agents, metal hip implants causing imaging artifacts, or a pacemaker.

\section{2 | Procedure}

Before the surgery, a contrast-enhanced CT abdomen with an arterial and an excretory phase was acquired. Combined with other relevant available preoperative images, a patient-specific 3D model was created manually using in-house developed software showing the tumor(s), arteries, veins, ureters, and bones. These segmentations were evaluated by the surgeon before surgery.

To track the patients' movement during surgery, three electromagnetic patient trackers (Philips, Best, the Netherlands) were attached to the skin at superficial bony structures of the patients' pelvis. As these tumors were relatively fixed with respect to the bone, the spatial position of the trackers is used to correct any patient motion during surgery. For patients enrolled before December 2015, these trackers were attached to the skin (and the locations were marked) just before the preoperative contrast-enhanced CT scan. In the OR, the trackers were repositioned on the skin according to the marked locations, and navigation was initiated. After December 2015, a hybrid OR containing a cone-beam CT (CBCT) was available, allowing to attach the patient trackers to the skin in the OR followed by a CBCT scan in surgical position. Subsequently, this CBCT scan was registered to the model where the navigation was initiated. This improved procedure increased the navigation accuracy significantly. ${ }^{6}$ In a previous study by Nijkamp et al., ${ }^{6}$ the accuracy (target registration error, TRE) of the abdominal navigation system was evaluated. In summary; the surgeon pointed out the physical locations of anatomical landmarks using the navigation pointer. These locations were stored compared with the same anatomical landmarks in the preoperative images. Depending on the patient position-that is, French or straight setup-, the TRE ranged from 4 to $6 \mathrm{~mm}$ using the CBCT scan. This is considered an adequate accuracy of the navigation system to localize anatomical structures. ${ }^{6}$

In-house created navigation software processes in real-time the tracker locations measured by the field-generator (NDI, Aurora Tabletop field-generator Northern Digital Inc.), which was positioned underneath the patient. Both the patients' model and the position of a sterile pointer (NDI) were visualized in 3D (see Figure 1), allowing the identification of critical anatomical structures or the tumor by the surgeon. In addition, any registered patients' scan can be displayed live in three orientations (coronal, sagittal, and transverse plane) with automatic visualization of arteries and bone. For more details, see Nijkamp et al. ${ }^{6}$ The time for setting up the tracking, positioning and intraoperative imaging was recorded starting from patient 6 .

\section{3 | Endpoints}

For our primary endpoint (feasibility), we assessed events regarding technical malfunctions of the system. The secondary outcomes were the safety and usability of the surgical navigation during these resections. Safety was evaluated based on adverse events caused by the system and the usability using a survey containing the System Usability Scale (SUS). ${ }^{7}$ The SUS consists of 10 items that can be answered by the surgeon based on a 5-point Likert scale (1-5) which are converted to a score ranging 0-100. Higher scores imply higher user-friendliness of the system and a mean SUS score above 70 is considered acceptable and indicates the system has a high chance of acceptance by users. ${ }^{8}$

TABLE 1 Patient characteristics

\begin{tabular}{|c|c|c|}
\hline & $N(\%)$ & $\begin{array}{l}\text { Median } \\
\text { (range) }\end{array}$ \\
\hline \multicolumn{3}{|l|}{ Gender } \\
\hline Male & $3(33)$ & \\
\hline Female & $6(66)$ & \\
\hline Age at surgery & & $63(46-74)$ \\
\hline Number of tumors per patient & & $1(1-3)$ \\
\hline \multicolumn{3}{|l|}{ Histology } \\
\hline Dedifferentiated liposarcoma (DDLS) & $3(33)$ & \\
\hline Gastrointestinal stromal tumor (GIST) & $2(22)$ & \\
\hline Leiomyosarcoma (LMS) & $1(11)$ & \\
\hline Myxofibrosarcoma (MFS) & $1(11)$ & \\
\hline $\begin{array}{l}\text { Malignant peripheral nerve sheath } \\
\text { tumor (MPNST) }\end{array}$ & $1(11)$ & \\
\hline Clear cell sarcoma (CCS) & $1(11)$ & \\
\hline \multicolumn{3}{|l|}{ Tumor type } \\
\hline Primary & $1(11)$ & \\
\hline Residual & $1(11)$ & \\
\hline Recurrent & $7(78)$ & \\
\hline \multicolumn{3}{|l|}{ Pretreatment } \\
\hline Radiotherapy & $3(33)$ & \\
\hline Systemic & $3(33)$ & \\
\hline None & $3(33)$ & \\
\hline
\end{tabular}




\subsection{Calculations and statistical analysis}

Tumor characteristics, distances to critical anatomical structures, and tumor area close to anatomical structures were calculated using the 3D models in Matlab (R2016a; MathWorks). High-risk tumor surfaces are only given for surfaces that have critical anatomical structures located at a distance of $<0.5 \mathrm{~cm}$ based on preoperative imaging.

Given the limited number of patients with accompanying nonnormal distributed data, outcomes will be given as median values with ranges. Differences between planned and actual surgery time were compared using a Wilcoxon signed-rank test using SPSS for Windows (Statistical Package for the Social Sciences, version 25; IBM statistics).

\section{3 | RESULTS}

\subsection{Patient and tumor characteristics}

We included nine intra-abdominal and pelvic STS patients with a total of twelve tumors, operated on between September 2015 and January 2020 (Table 1). Two of the patients were operated on before December 2015 (patients 1 and 2) and therefore registration of the patient trackers was slightly different in these cases as discussed in Section 2. The most common histological STS subtype was dedifferentiated liposarcoma. The median age at surgery was 63 years (range 46-72). Seven patients had (a) recurrent tumor(s) (78\%). In 3 of 9 patients, neoadjuvant radiotherapy and in 3 other patients neoadjuvant systemic treatment preceded the surgery.

The median tumor size was $4.6 \mathrm{~cm}$ (range 2.4-10.0) and median volume $10.5 \mathrm{ml}$ (range 1-86). The tumor characteristics, distances from tumor to critical anatomical structures, and surgery outcomes are shown in Table 2 and Figure 2. For illustration, the pointer usage over time during surgery in relation to the tumor is shown for a single case in Figure 1.

\section{2 | Feasibility}

The surgical navigation system could be used in all cases and had no technical malfunctions. Extra operation room (OR) time due to surgical navigation consisted of two different activities: (A) Placing the patient trackers and the positioning of the patient required a median time of $1.5 \mathrm{~min}$ (range $0-8$ ). (B) The intraoperative imaging by $\mathrm{CBCT}$ required a median time of $8.5 \mathrm{~min}$ (range 7-12). In total, setting up navigation added a median time of $10 \mathrm{~min}$ (range 7-20).

The median surgery time was $142 \mathrm{~min}$ (range 78-241), whereof median $98 \mathrm{~min}$ (range 27-197) the navigation was used. The pointer was used over a median of 7.4 min (range 3.8-19.4), equal to a median of $10 \%$ of time relative to availability of the navigation system (range $4 \%-31 \%$ ).

\section{3 $\quad$ Critical anatomical structures}

We identified which critical anatomical structures were located closer than $0.5 \mathrm{~cm}$ from the tumor border. The median shortest distance from the tumor to a major artery was $0.0 \mathrm{~cm}$ (range $0.0-1.9$ ), to a major vein $0.2 \mathrm{~cm}$ (range 0.0-6.0), to a bony structure $0.9 \mathrm{~cm}$ (range 0.0-2.3) and to ureter $0.7 \mathrm{~cm}$ (range 0.0-6.6), see Table 2. The median high-risk tumor surface is visualized in Figure 2 as well. Patient/tumor 5 had the lowest "high-risk tumor surface" (equal to $0 \%$ ), with a ureter at $0.44 \mathrm{~cm}$ distance.

\section{4 | Usability}

We have collected five surveys completed by different surgeons. All surgeons scored a SUS of 90 , which classifies the surgical navigation system as a superior system and indicates high user-friendliness, see Figure 3. All respondents stated that navigation resulted in subjective shorter surgery time and it made the resections easier and safer due to better localization of the tumor in all cases. Navigation contributed to the assurance of decisions and actions during the surgery. The only question not in favor of the navigation system indicates that technical support is required to utilize the system. On the questions comparing navigation with the conventional setting, the surgical navigation scored better, especially on localization of the tumor, complications, and total surgery time. All surgeons indicate they would use the surgical navigation system again and that the advantages of using the system were worth the extra setup time.

\subsection{Safety and oncological outcome}

No adverse events have occurred regarding the navigation system and only one patient had a non-navigation-related complication >grade II according to the Clavien-Dindo classification. ${ }^{9}$ This patient required surgical drainage of a presacral abscess. Median blood loss during surgery was $100 \mathrm{ml}$ (range 20-1050). The median length of hospital stay was 6 days (range 3-10), and $89 \%$ of the resections were R0, see Table 2. Median follow-up after surgery was 37 months (range 4-51). Local recurrence occurred in four patients (44\%), for all patients this was their second recurrence. Mean recurrence-free survival was 31 months $(95 \% \mathrm{Cl}$ 16.5-45.3). Median recurrence-free survival was not reached. One patient died of recurrent disease.

\section{4 | DISCUSSION}

In this study, we show that this novel electromagnetic surgical navigation technique is a feasible system for resecting intra-abdominal or pelvic STS, given the absence of technical malfunctions and lack of adverse events or navigation-related complications in all patients. 
TABLE 2 Tumor characteristics, distance from tumor to critical anatomical structures and surgery outcomes

\begin{tabular}{|c|c|c|c|c|c|c|c|c|c|c|c|c|c|}
\hline Patient (tumor) & $\begin{array}{l}1 \\
(1)\end{array}$ & (2) & (3) & $\begin{array}{l}2 \\
-\end{array}$ & $\begin{array}{l}3 \\
-\end{array}$ & $\begin{array}{l}4 \\
-\end{array}$ & $\begin{array}{l}5 \\
-\end{array}$ & $\begin{array}{l}6 \\
(1)\end{array}$ & (2) & $\begin{array}{l}7 \\
-\end{array}$ & $\begin{array}{l}8 \\
-\end{array}$ & $\begin{array}{l}9 \\
-\end{array}$ & $\begin{array}{l}\text { Median } \\
\text { (range) }\end{array}$ \\
\hline \multicolumn{14}{|l|}{ Tumor characteristics } \\
\hline Volume (ml) & 16 & 7 & 11 & 4 & 10 & 1 & 19 & 7 & 14 & 10 & 86 & 15 & $10.5(1-86)$ \\
\hline Veins (cm) & 0.3 & $>5$ & 3.0 & 0.1 & $<0.1$ & $<0.1$ & 1.1 & $<0.1$ & $<0.1$ & 2.7 & $<0.1$ & 1.8 & $0.2(0.0-6.0)$ \\
\hline Bone $(\mathrm{cm})$ & 0.2 & 0.8 & $<0.1$ & 2.3 & 0.4 & 1.7 & 2.3 & 1.8 & 0.9 & $<0.1$ & $<0.1$ & 1.9 & $0.9(0.0-2.3)$ \\
\hline Ureter $(\mathrm{cm})$ & 3.0 & $>5$ & $>5$ & 4.5 & $<0.1$ & 0.3 & 0.4 & $<0.1$ & 0.9 & $>5$ & 0.6 & 1.8 & $0.7(0.0-6.6)$ \\
\hline Hospitalization (days) & & 8 & & 6 & 8 & 10 & 8 & \multicolumn{2}{|l|}{5} & 3 & 6 & 3 & $6(3-10)$ \\
\hline Vascular damage & & No & & No & No & No & No & \multicolumn{2}{|c|}{ Yes } & No & No & No & - \\
\hline Removing periosteum & & No & & No & No & No & No & \multicolumn{2}{|c|}{ No } & Yes & Yes & No & - \\
\hline Ureter damage & & No & & No & No & No & No & \multicolumn{2}{|c|}{ No } & No & No & No & - \\
\hline Surgery time (min) & & 170 & & 113 & 130 & 142 & 235 & \multicolumn{2}{|c|}{147} & 112 & 241 & 78 & $142(78-241)$ \\
\hline Navigation available ( $\mathrm{min}$ ) & & 95 & & 27 & 52 & 102 & 154 & \multicolumn{2}{|c|}{156} & 98 & 197 & 62 & $98(27-197)$ \\
\hline Pointer usage (min) & & - & & 8.3 & 5.4 & 3.8 & 7.6 & \multicolumn{2}{|c|}{7.4} & 19.4 & - & 6.9 & $7.4(3.8-19.4)$ \\
\hline Pointer usage $(\%)^{b}$ & & - & & 31 & 10 & 4 & 5 & \multicolumn{2}{|l|}{5} & 20 & - & 13 & $10(4-31)$ \\
\hline
\end{tabular}

${ }^{\mathrm{a}} \mathrm{RO}$ is for the dedifferentiated part of the tumor (liposarcoma).

${ }^{b}$ Pointer usage (\%) is the percentage of time relative to availability of the navigation system.

As the majority of the tumors were recurrent (78\%) and/or were resected after neoadjuvant therapy (66\%), almost all tumors were located in pretreated areas with fibrosis and anatomical changes. Despite these challenging circumstances, also indicated by the close proximity of the tumors to critical anatomical structures, all tumors were successfully localized with the use of navigation and safely resected without damaging any critical anatomical structure.

The technique was not very time-consuming: the preparations took an additional $10 \mathrm{~min}$ OR time. Adding the navigation does not seem to extend the surgery, as the median operation time (142 min, range 78-241) with navigation was below the historical time of similar surgeries within our institute of $180 \mathrm{~min}$. In addition, the navigation preparation time has shortened compared to the previous study by Nijkamp et al., ${ }^{6}$ from 18 to $10 \mathrm{~min}$. The additional time for using navigation does not include the creation of the $3 \mathrm{D}$ model. This depends on the experience of the imaging specialist and the complexity of the case, which varies between 1 and $3 \mathrm{~h} .{ }^{6}$ However, 3D model creation takes place before the surgery and has no effect on costly OR time. With deep learning developments in image processing, it can be expected that $3 \mathrm{D}$ model creation will be fully automated and within minutes.

The pointer is used during a median $10 \%$ (range $4 \%-31 \%$ ) of time relative to the availability of the navigation system, suggesting the relative need for the surgeons to have assistance in localizing and safely removing the tumor. This is illustrated by the questionnaire results, where surgeons indicated high usability of the navigation system with positive responses of the participating surgeons.

Surgeons also indicated in this questionnaire that there were potentially more negative resection margins by using the surgical navigation system, although, the current navigation system is not designed to determine microscopically clear margins. However, a recent publication showed a significant increase in $\mathrm{RO}$ resections 


\section{Patient 1 \\ Tumor 1 \\ Volume $=16 \mathrm{ml}$ \\ $\mathrm{D}=2.1 \times 2.4 \times 6.9 \mathrm{~cm}$}
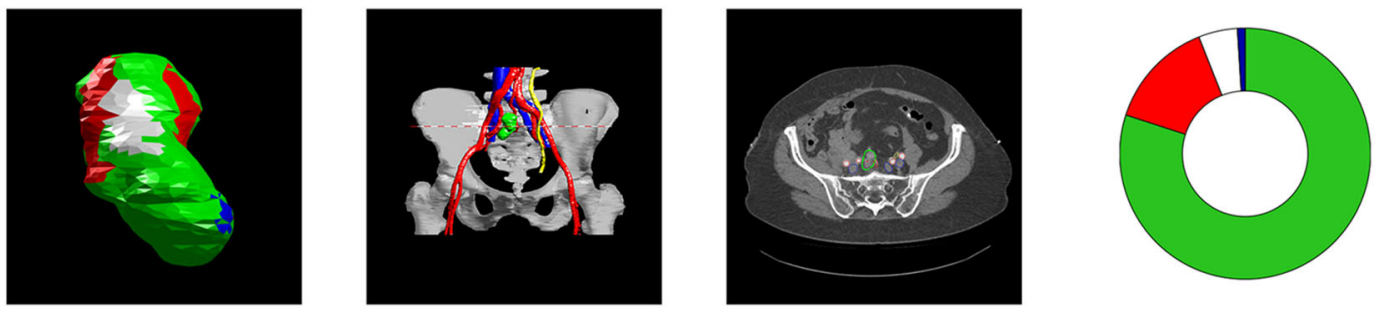

\section{Patient 1}

Tumor 2

Volume $=7 \mathrm{ml}$

$\mathrm{D}=1.2 \times 2.7 \times 5.9 \mathrm{~cm}$
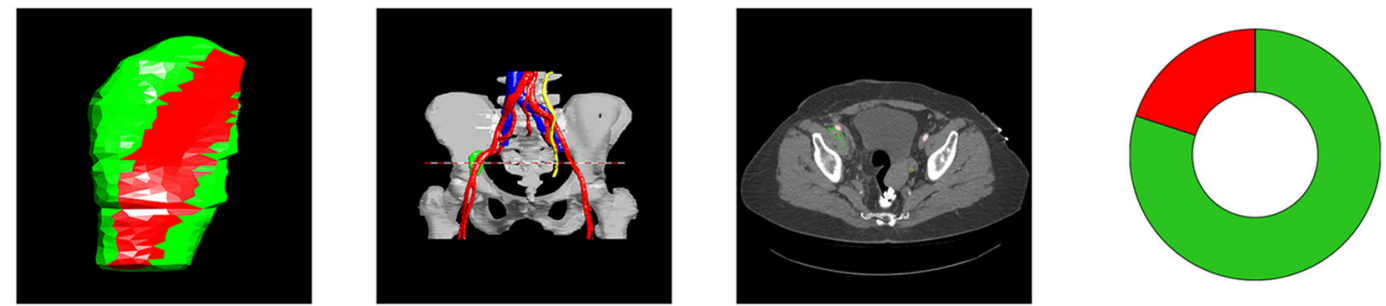

\section{Patient 1}

Tumor 3

Volume $=11 \mathrm{ml}$ $D=1.6 \times 2.7 \times 7.2 \mathrm{~cm}$
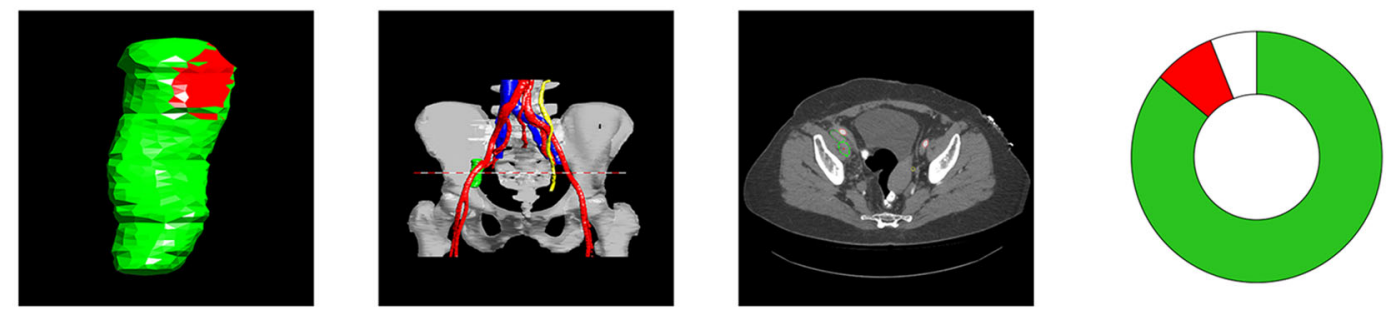

\section{Patient 2}

Volume $=4 \mathrm{ml}$ $\mathrm{D}=1.6 \times 1.9 \times 3.1 \mathrm{~cm}$
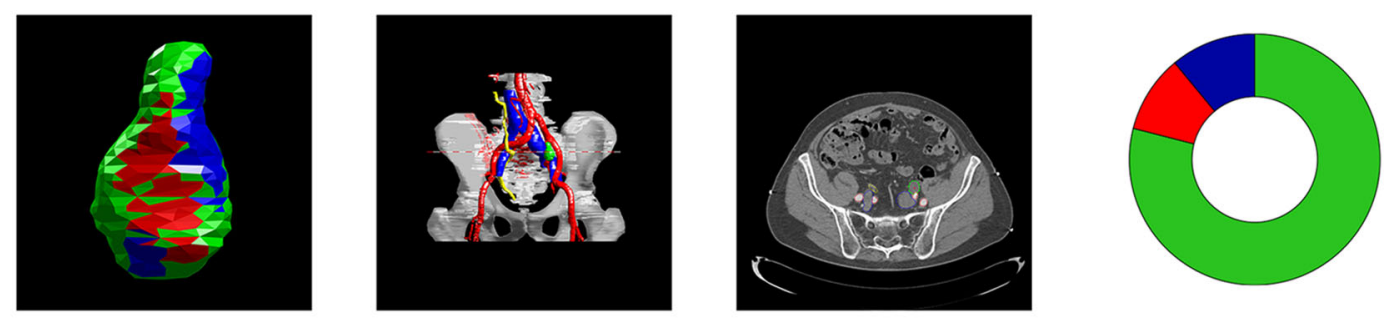

Patient 3
Volume $=10 \mathrm{ml}$
$D=1.7 \times 3 \times 4.1 \mathrm{~cm}$
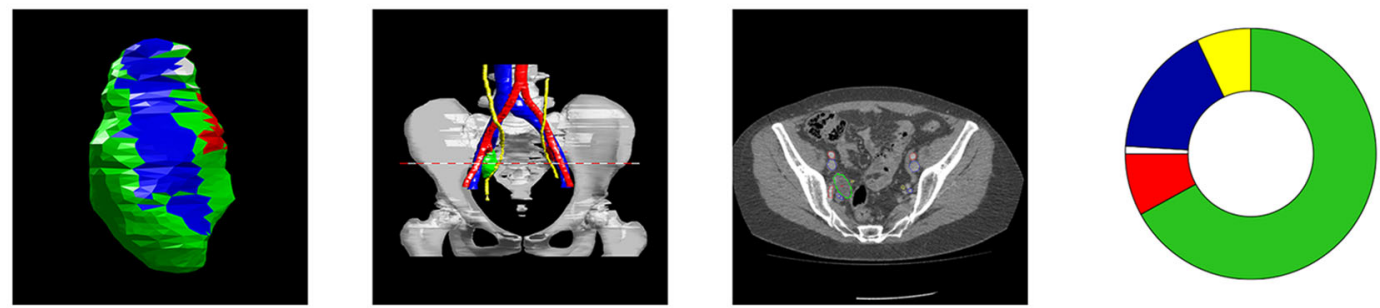

\section{Patient 4}

Volume $=1 \mathrm{ml}$

$\mathrm{D}=0.9 \times 1.2 \times 2.4 \mathrm{~cm}$

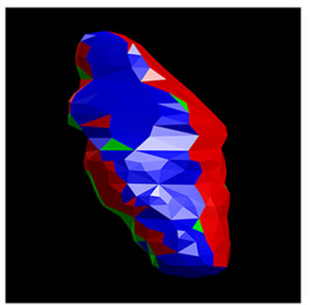

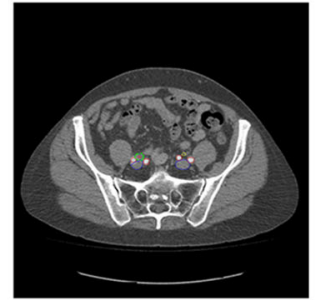

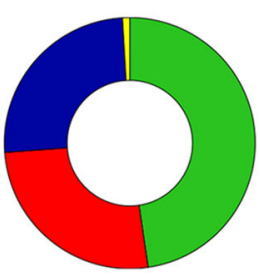

FIGURE 2 Tumor characteristics and nearby critical anatomical structures (per patient from left to right). A three-dimensional (3D) model of each tumor with color-coded nearby $(<0.5 \mathrm{~cm})$ critical anatomical structures, a 3D model of the tumor(s) in relation to critical anatomical structures, and a computed tomography (CT) scan of the patient with tumor(s) and critical anatomical structures highlighted. The right column shows the total tumor surface and which percentage is "at risk" due to nearby $(<0.5 \mathrm{~cm})$ critical anatomical structures. Tumor (not at risk), green; artery, red; vein, blue; ureter, yellow; bone, white 


\section{Patient 5 \\ Volume $=19 \mathrm{ml}$ \\ $\mathrm{D}=2.4 \times 3.5 \times 5 \mathrm{~cm}$}
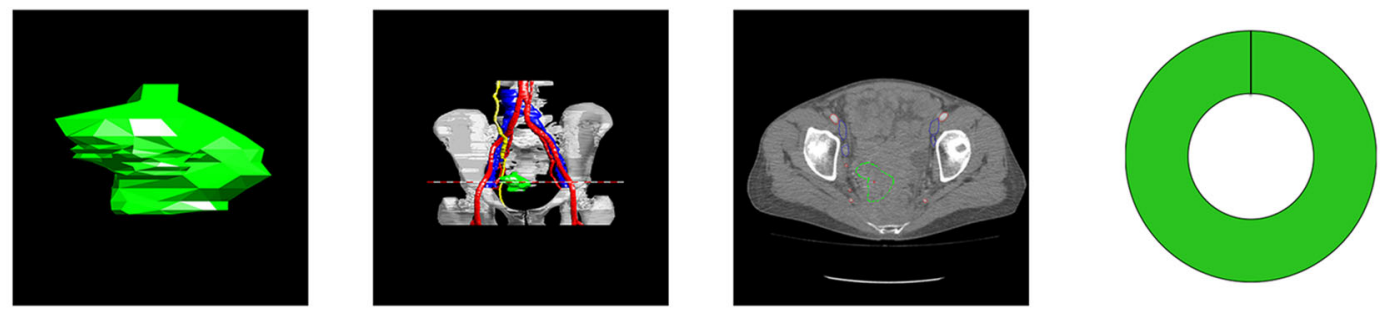

\section{Patient 6 \\ Tumor 1 \\ Volume $=7 \mathrm{ml}$ \\ $\mathrm{D}=1.7 \times 2 \times 5.3 \mathrm{~cm}$}
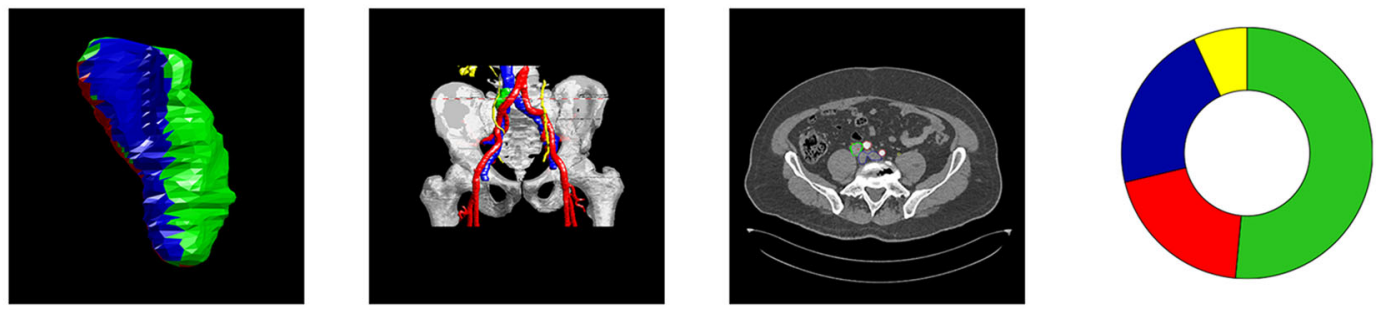

Patient 6

Tumor 2

Volume $=14 \mathrm{ml}$

$\mathrm{D}=2.1 \times 3.4 \times 3.9 \mathrm{~cm}$
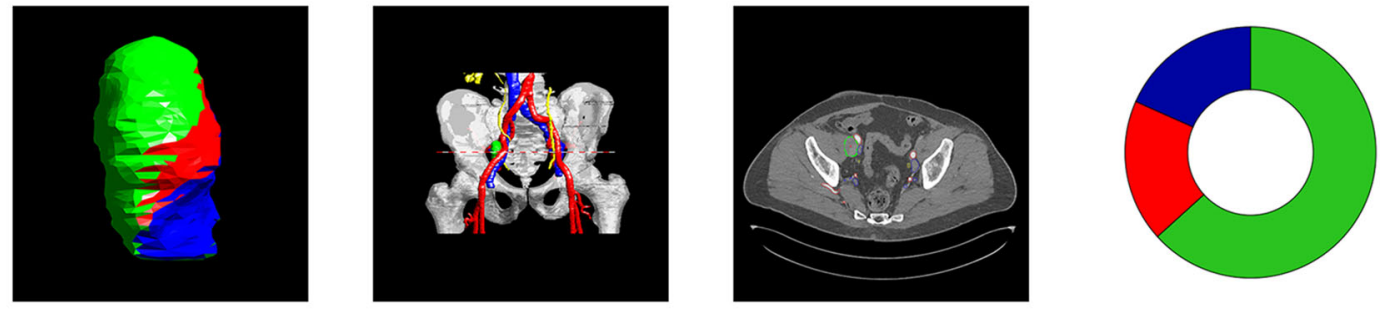

\section{Patient 7}

Volume $=10 \mathrm{ml}$

$D=1.4 \times 2.6 \times 8.1 \mathrm{~cm}$
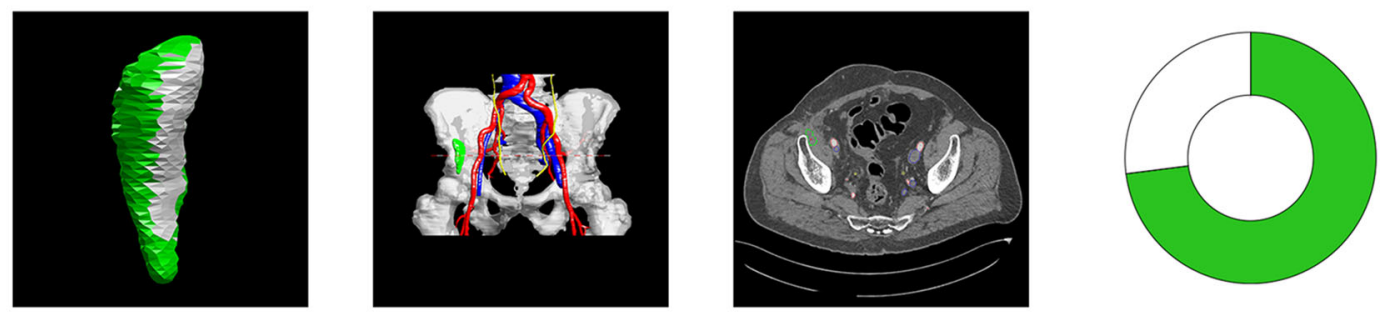

Patient 8
Volume $=86 \mathrm{ml}$
$D=2.8 \times 7 \times 10.4 \mathrm{~cm}$
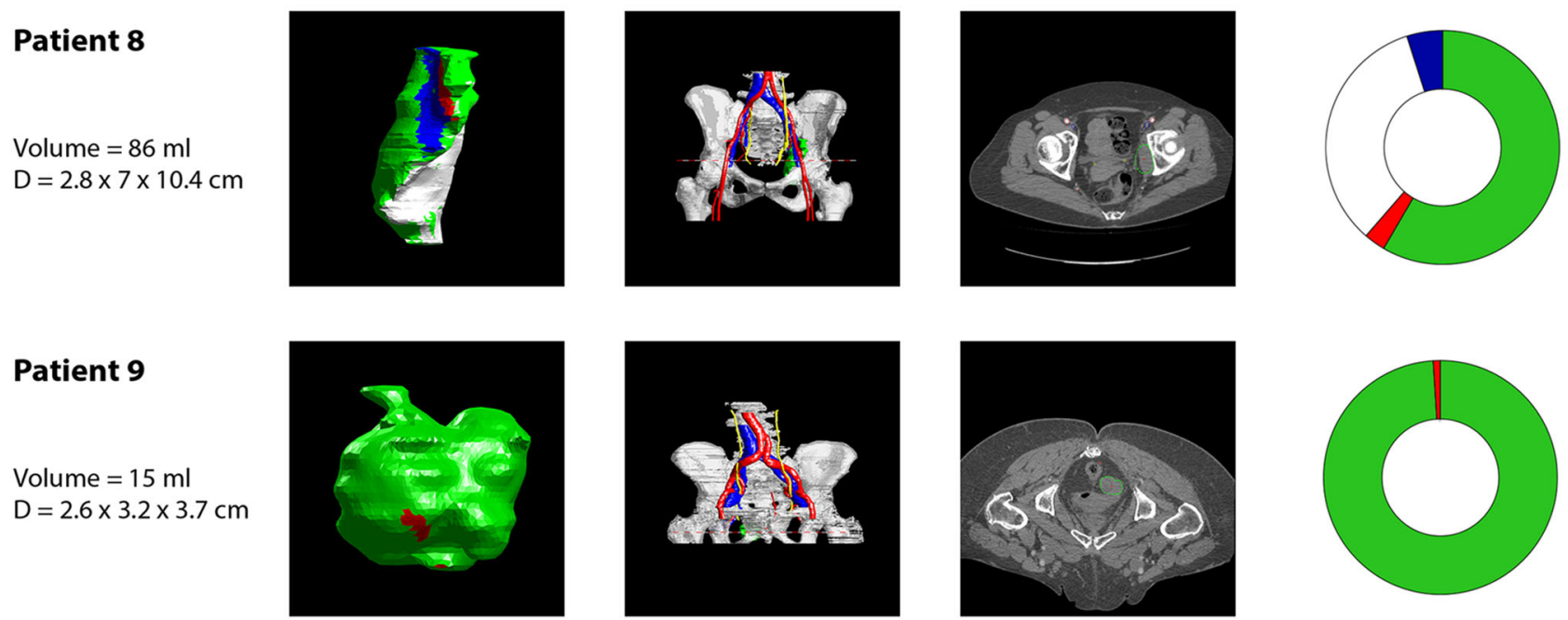

FIGURE 2 Continued 


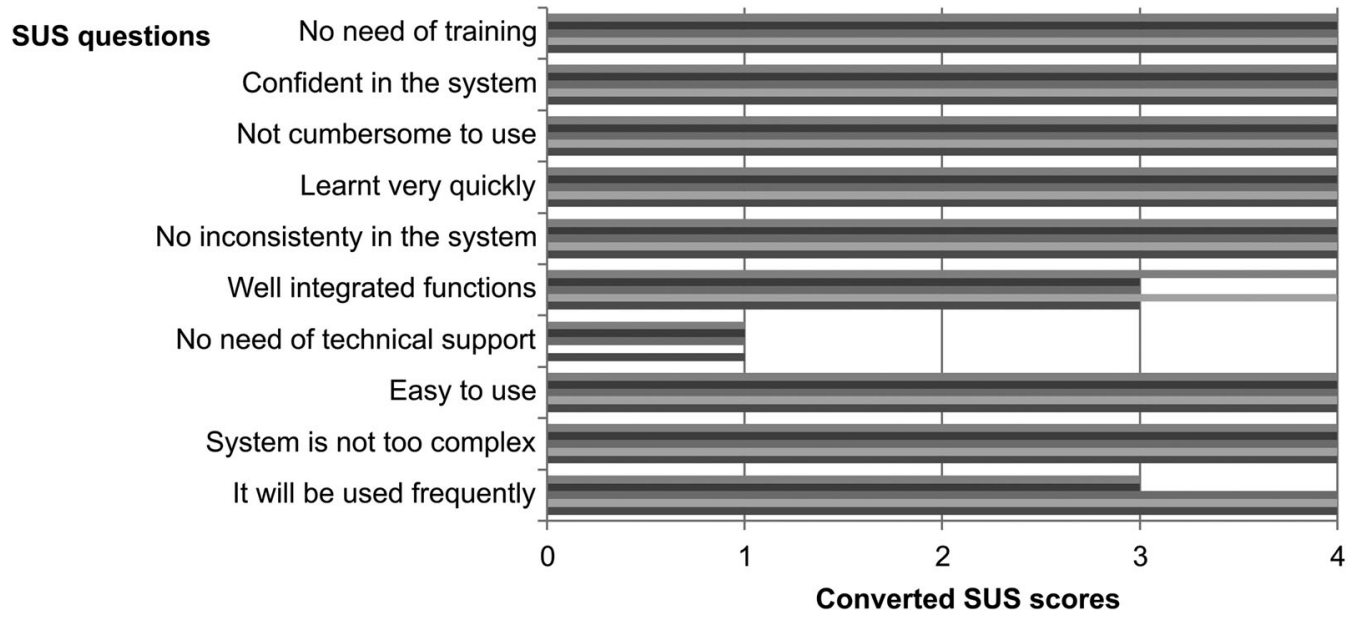

FIGURE 3 Converted System Usability Scale (SUS) scores per respondent per question. Five respondents, ten questions. $0=$ strongly disagree, 4 = strongly agree

when using electromagnetic navigation for recurrent rectal cancer surgery, ${ }^{5}$ possibly secondary to the use of the navigation system.

Although navigation is already widely used with good results in some other surgical fields with highly rigid structures or tumors such as neurosurgery and orthopedics, ${ }^{10,11}$ surgical navigation for STS is relatively new. Several surgical navigation systems have shown promising results for bone sarcomas, ${ }^{12-14}$ but for STS, and especially intra-abdominal or pelvic STS, the literature on the application of navigation techniques is very limited. ${ }^{15,16}$ Electromagnetic navigation is not the only navigation technology developed. Optical tracking uses cameras and markers on surgical instruments instead of an electromagnetic field. ${ }^{15}$ This requires a direct view of the used instruments, which is not possible when operating in the pelvis. Fluorescence-guided surgery, as described by Vahrmeijer et al. and Tipirneni et al., uses invisible near-infrared (NIR) fluorescent light in combination with fluorescent contrast agents. ${ }^{16-18}$ This technique visualizes the tumor without ionizing radiation exposure or alterations of the surgical field but is only of added value if you are already in very close proximity of the tumor due to limited tissue penetration. Recently, a phase-1 single-center study has deemed the fluorescent contrast agent bevacizumab-800CW feasible and safe for intra-operative imaging of STS. ${ }^{16}$ Also other optical techniques, such as Diffuse Reflectance Spectroscopy, can be used to visualize tumor tissue. Diffuse Reflectance Spectroscopy (DRS) detects the composition of tissue by measuring the interaction between light and tissue without the need for exogenous agents. The technique has already been successfully evaluated in multiple oncological domains for discriminating tumor tissue from healthy tissue with classification accuracies of $0.86 . .^{19,20}$ Our study with electromagnetic navigation is especially useful in cases where finding the tumor and identification of critical structures is challenging, but to define tumor margins more accurately, this technique could be combined with the optical tissue sensing by DRS or fluorescence-guided technique.

The study as presented here has some limitations, like the number of patients we were able to include over time due to the low incidence of
STS. In addition, this study only included patients scheduled for laparotomy. The combination of these two factors makes is difficult to prove the added value of surgical navigation for this specific (tumor) group with sufficient patients in a future randomized controlled trial. Thirdly, two out of six surgeons were involved in the development of the navigation technique, which is essential for an intuitive setup and workflow. However, these two surgeons were only involved in one of the presented cases. Obviously, the current expansion of this technique to other hospitals will allow further assessment of the functional and clinical usability of abdominal navigation. Another limitation is the fact that the navigation system has an accuracy to point exactly at an anatomical landmark in the preoperative image of up to $4-6 \mathrm{~mm}^{6}$ which prevents using the navigation system for evaluation microscopic margins and affects the reliability of the calculated distances of the tumor to critical anatomical structures as presented in Table 2. However, these distances do reflect the "challenging" element of the resection due to close relations, rather than precise measures. This system is only applicable in relatively rigid tumor (s) to ensure the accuracy of the system. This means that as the resection progresses and the tumor becomes more mobile, the error margin could increase. However, this can be improved by adding accurate real-time tumor tracking as published recently. ${ }^{4}$ The final limitation is the lack of major nerves delineations, which could be improved by adding an MRI with a special nerve sequence to the preoperative imaging scheme.

The added value of this technique is the combination of localizing the tumor and strengthening the awareness of critical anatomical structures nearby, minimizing the often uncertain and comprehensive exploratory surgeries. Whether surgical navigation by itself is indeed leading to shorter OR time, fewer complications, and better resection margins (therefore possibly a better oncological outcome) compared to conventional surgery is hard to prove, for this more comparative studies will be needed to use this technique as the standard of care for challenging tumors.

Future prospects could be to further analyze how the surgeon uses the navigation in detail by recording exactly when and for which purpose the navigation is used. If we would track this, we would be 
able to display the navigation information in a more intuitive way, tailored to the structure of interest. However, this study does show that there is potential for novel navigation techniques possibly improving surgical quality for sarcoma in specific cases.

\section{5 | CONCLUSION}

In conclusion, electromagnetic surgical navigation assisted in the resection of challenging STS cases without technical or safety issues. This technique can be used in the resection of smaller, relatively rigid recurrent and/or pretreated intra-abdominal or pelvic STS, closely related to critical anatomical structures, to improve surgical quality.

\section{ACKNOWLEDGMENTS}

We would like to thank Koningin Wilhelmina Fonds-Alpe d'HuZes (NKI 2014-6596) for their funding.

\section{CONFLICT OF INTERESTS}

The authors declare that there are no conflicts of interest.

\section{DATA AVAILABILITY STATEMENT}

The data that support the findings of this study are available from the corresponding author upon reasonable request.

\section{ORCID}

\section{Sophie J. M. Reijers (D) http://orcid.org/0000-0003-4872-7746}

\section{REFERENCES}

1. WHO Classification of Tumours, Soft Tissue and Bone Tumours. Vol 3, 5th ed. WHO Classification of Tumours Editorial Board; 2020.

2. Brennan MF, Antonescu CR, Moraco N, Singer S. Lessons learned from the study of 10,000 patients with soft tissue sarcoma. Ann Surg. 2014;260(3):416-421, discussion 421-412.

3. Mullen JT, van Houdt W. Soft tissue tumors of the pelvis: technical and histological considerations. J Surg Oncol. 2018;117(1):48-55.

4. Kok END, Eppenga R, Kuhlmann KFD, et al. Accurate surgical navigation with real-time tumor tracking in cancer surgery. NPJ Precis Oncol. 2020;4:8.

5. Kok END, van Veen R, Groen HC, et al. Association of image-guided navigation with complete resection rate in patients with locally advanced primary and recurrent rectal cancer: a nonrandomized controlled trial. JAMA Netw Open. 2020;3(7):e208522.

6. Nijkamp J, Kuhlmann KFD, Ivashchenko O, et al. Prospective study on image-guided navigation surgery for pelvic malignancies. J Surg Oncol. 2019;119(4):510-517.
7. Brooke JB. SUS-A quick and dirty usability scale. 1996.

8. Bangor AKP, Miller JT. An empirical evaluation of the system usability scale. Int J Hum Comput Interact. 2008;24(24):574-594.

9. Dindo D, Demartines N, Clavien PA. Classification of surgical complications: a new proposal with evaluation in a cohort of 6336 patients and results of a survey. Ann Surg. 2004;240(2):205-213.

10. Kochanski RB, Lombardi JM, Laratta JL, Lehman RA, O'Toole JE. Image-guided navigation and robotics in spine surgery. Neurosurgery. 2019;84(6):1179-1189.

11. Mavrogenis AF, Savvidou OD, Mimidis G, et al. Computer-assisted navigation in orthopedic surgery. Orthopedics. 2013;36(8):631-642.

12. Cho HS, Oh JH, Han I, Kim HS. The outcomes of navigation-assisted bone tumour surgery: minimum three-year follow-up. J Bone Joint Surg Br. 2012;94(10):1414-1420.

13. Abraham JA, Kenneally B, Amer K, Geller DS. Can navigationassisted surgery help achieve negative margins in resection of pelvic and sacral tumors? Clin Orthop Relat Res. 2018;476(3):499-508.

14. Jeys L, Matharu GS, Nandra RS, Grimer RJ. Can computer navigation-assisted surgery reduce the risk of an intralesional margin and reduce the rate of local recurrence in patients with a tumour of the pelvis or sacrum? Bone Joint J. 2013;95-B10: 1417-1424

15. Reijnders $\mathrm{K}$, Coppes $\mathrm{MH}$, van Hulzen AL, Gravendeel JP, van Ginkel RJ, Hoekstra HJ. Image guided surgery: new technology for surgery of soft tissue and bone sarcomas. Eur J Surg Oncol. 2007; 33(3):390-398.

16. Steinkamp PJ, Pranger BK, Li MF, et al. Fluorescence-guided visualization of soft-tissue sarcomas by targeting vascular endothelial growth factor a: a phase 1 single-center clinical trial. J Nucl Med. 2021;62(3):342-347.

17. Vahrmeijer AL, Hutteman M, van der Vorst JR, van de Velde CJ, Frangioni JV. Image-guided cancer surgery using near-infrared fluorescence. Nat Rev Clin Oncol. 2013;10(9):507-518.

18. Tipirneni KE, Warram JM, Moore LS, et al. Oncologic procedures amenable to fluorescence-guided surgery. Ann Surg. 2017;266(1): 36-47.

19. de Boer LL, Molenkamp BG, Bydlon TM, et al. Fat/water ratios measured with diffuse reflectance spectroscopy to detect breast tumor boundaries. Breast Cancer Res Treat. 2015;152(3):509-518.

20. Baltussen EJM, Brouwer de Koning SG, Sanders J, et al. Tissue diagnosis during colorectal cancer surgery using optical sensing: an in vivo study. J Transl Med. 2019;17(1):333.

How to cite this article: Reijers SJM, Heerink WJ, Van Veen $\mathrm{R}$, et al. Surgical navigation for challenging recurrent or pretreated intra-abdominal and pelvic soft tissue sarcomas. J Surg Oncol. 2021;124:1173-1181. https://doi.org/10.1002/jso.26624 